\title{
Neurosciences Community Unites to Promote Increased Awareness
}

Montreal (Thursday, June 18, 1998) The Canadian Congress of Neurological Sciences (CCNS) announced a partnership formed to create awareness of neurological disorders, such as Alzheimer's Disease, Stroke and Epilepsy, at the Bonaventure Hilton. The coalition is known as the CCNS Partners Coalition.

Canadian Congress of Neurological Sciences Executive, Vice President, Rick Riopelle says, "Both the economic and personal burdens of neurological disorders demonstrate the great need to reduce the incidence of neurological disease."

The burden of illness in Canada for neurological disorders is staggering. In Canada alone, neural injury and disease strike more than four million people, this represents 1 in 7.5 persons and costs Canadians $\$ 30$ billion/year. For most of these conditions, treatment is still either unsatisfactory or non-existent.

In fact, more than 1,000 disorders of the brain and the nervous system result in more hospitalizations than any other disease group, including heart disease and cancer.

The CCNS partners are comprised of the Canadian Association of Child Neurology, Canadian Neurological Society, Canadian Neurosurgical Society, the Canadian Society of Clinical Neurophysiologists, the Canadian Association for Neuroscience, patient care groups and the private sector. They are committed to working together to raise awareness of neurological disorders, to promote and support research and education relevant to health and disease, and to provide costeffective health services to patients and their caregivers.

The CCNS is a non-profit organization dedicated to the advancement of knowledge, patient care, and collegiality among health care professionals and research scientists working in all branches of neurological sciences.

\section{La communauté des sciences neurologiques se solidarise en vue de favoriser une augmentation du financement}

Montréal (Jeudi 18 juin 1998) - Le congrès canadienne des sciences neurologiques (CCSN) a annoncé au Bonaventure Hilton un partenariat formé dans le but de répondre aux besoins de financement de projets de recherche en neurologie, tels que la recherche sur la maladie d'Alzheimer. La coalition est connue sous le nom Partenariat du CCSN.

Le vice-président directeur du Congrés canadien des sciences neurologiques, Rick Riopelle, déclare Les fardeaux économique et personnel des troubles neurologiques prouvent le grand besoin de réduire la fréquence de l'affection neurologique.

Le fardeau des troubles neurologiques au Canada est renversant. Au Canada uniquement, les lésions et maladies neurales atteignent plus de quatre millions de personnes, ce qui correspond à une personne parmi 7,5 et coûte aux Canadiens 30 milliards de dollars par année. Pour la plupart de ces troubles, le traitement est insatisfaisant ou inexistant.

De fait, plus de 1000 troubles du cerveau et du système nerveux entraînent plus d'admissions à l'hôpital que tout autre groupe de maladies, y compris la maladie du coeur et le cancer.

Les associés du CCSN comprennent l'Association canadienne de neurologie pédiatrique, la Société canadienne de neurologie, la Société canadienne de neurochirurgie, la Société canadienne de neurophysiologie clinique, l'Association canadienne des neurosciences, des groupes de soins et le secteur privé. Ils sont déterminés à travailler en collaboration dans le but de sensibiliser le public aux troubles neurologiques, de favoriser et díappuyer la recherche et l'éducation liées à la santé et la maladie, et d'offrir des services de santé rentables aux patients et aux fournisseurs de soins.

Le CCSN est une organisation à but non lucratif qui se consacre au progrès des connaissances, des soins aux patients et de la collégialité chez les professionnels de la santé et les chercheurs scientifiques oeuvrant dans tous les secteurs des sciences neurologiques. 